\title{
Graded-Index Plastic Optical Fiber with High Mechanical Properties Enabling Easy Network Installations. II
}

\author{
Takaaki Ishigure, ${ }^{1}$ Miki Hirai, ${ }^{2}$ Masataka Sato, ${ }^{2}$ Yasuhiro Koike ${ }^{1}$ \\ ${ }^{1}$ Faculty of Science and Technology, Keio University, 3-14-1, Hiyoshi, Kohoku-ku, Yokohama 223-8522, Japan \\ ${ }^{2}$ Japan Science and Technology Corporation, ERATO, 144-8, Ogura, Saiwai-ku, Kawasaki 212-0054, Japan
}

Received 25 November 2002; accepted 10 April 2003

\begin{abstract}
It was shown how high mechanical strength should be provided in the high numerical aperture (NA) graded-index plastic optical fiber (GI POF). In this newly developed GI POF, a copolymer of methyl methacrylate (MMA) and 2,2,2-trifluoroethyl methacrylate (3FMA) was used to increase the NA. The GI POF we proposed previously was composed of a PMMA homopolymer cladding and a doped PMMA core. It was previously shown that substituting the copolymer P(MMA-3FMA) for the PMMA as the cladding material made little change in the fiber's light-transmission characteristics. This study focused on mechanical flexibility, which is one of the most important ad-
\end{abstract}

vantages of the POF. It was found that the P(MMA-3FMA)clad GI POF had almost the same or superior mechanical strength in addition to the excellent light-propagation characteristics. It was also found that such excellent mechanical properties were achieved using a small dopant concentration and optimum heat-drawing conditions. (c) 2003 Wiley Periodicals, Inc. J Appl Polym Sci 91: 410-416, 2004

Key words: mechanical properties; refractive index; plastic optical fibers; poly(methyl methacrylate) (PMMA); light transmission

\section{INTRODUCTION}

The advantages of optical fiber technologies in data communications area became apparent in the early stages of their development. With the maturity of optoelectronic technology in terms of level of integration, cost, and reliability, development activity began in the 1990s to apply the lightwave technology to data communications. Single-mode silica fibers are widely used in long-distance telecommunications networks, whereas many of short-distance data links favor the less-expensive multimode fiber technology. With the growing demand for high-speed accessing to the Internet, data rates higher than a gigabit are required, even for such short-distance data communications areas.

We have proposed a high-bandwidth graded-index plastic optical fiber (GI POF) as a promising candidate for the high-speed data transmission medium. ${ }^{1,2}$ One of the largest advantages of the POF should be an excellent mechanical flexibility even if it has a large diameter, such as several hundred micrometers to 1 $\mathrm{mm}$, compared to the silica optical fibers. Therefore, the GI POF mechanically enables a smaller bending radius than silica-based optical fibers, by which the installation cost of a GI POF network is substantially reduced. This is one of the reasons that a POF network is expected to be used in the home and office.

Correspondence to: T. Ishigure (ishigure@appi.keio.ac.jp).

Journal of Applied Polymer Science, Vol. 91, 410-416 (2004) (C) 2003 Wiley Periodicals, Inc.
Regarding the mechanical flexibility of a poly(methyl methacrylate) (PMMA)-based GI POF, we reported in our previous studies ${ }^{3,4}$ that by providing the appropriate polymer-chain orientations in the fiber axial direction, the GI POF with almost the same mechanical properties as the commercially available stepindex (SI) POF could be obtained.

On the other hand, the minimum bending radius of the fiber in optical data links should be determined not only by the mechanical issue but also by the optical bending loss. We proposed a high numerical aperture (NA) GI POF to decrease the bending loss by introducing a copolymer of a partially fluorinated polymer [2,2,2-trifluoroethyl methacrylate (3FMA)] and PMMA to the cladding. ${ }^{4}$ Use of the copolymer P(MMA-3FMA) allowed the cladding to have a lower refractive index than that of the PMMA homopolymer cladding, which realized a low bending loss.

This study focuses on the mechanical flexibility of both the PMMA-clad and P(MMA-3FMA)-clad GI POFs. If the copolymer-clad GI POF could have a sufficiently high mechanical flexibility, it becomes a more suitable candidate for the high-speed physical medium in the forthcoming broadband home and office networks.

\section{EXPERIMENTAL}

\section{Fabrication of GI POF}

We proposed the interfacial-gel polymerization method to obtain a GI preform rod having a diameter 


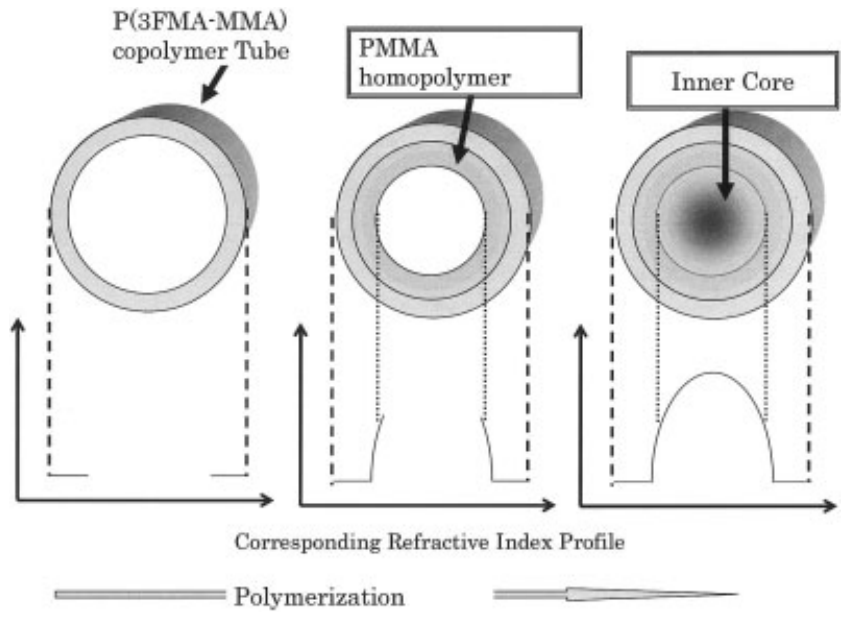

Figure 1 Schematic representation of the two-step interfacial-gel polymerization technique.

of $18-30 \mathrm{~mm} \cdot{ }^{1,2,5}$ To form the core region of the GI POF by the interfacial-gel polymerization process, the mixture of the monomer and an aromatic dopant was injected into a polymer tube having an $18-30-\mathrm{mm}$ outer diameter, and the tube was heated from the surrounding environment to polymerize the core region. During this process, the inner wall of the tube is slightly dissolved and swollen by the monomer mixture and forms a polymer-gel phase. This polymergel phase plays an important role in forming the concentration distribution of the dopant, which is related to the refractive index distribution. ${ }^{5}$

In the case of the P(MMA-3FMA)-clad GI POF, an excess scattering loss is a concern after the core polymerization because of the poor miscibility between the cladding materials (copolymer of MMA and 3FMA) and the aromatic dopant. If the MMA-dopant mixture is directly injected into the copolymer tube and polymerized (i.e., the same way as in the interfacial-gel polymerization technique) the aromatic dopant must diffuse into the copolymer gel phase. Because of the poor miscibility between the aromatic compound and the MMA-3FMA copolymer, large heterogeneities are caused, which induces an excess scattering loss. Thus, a low-loss GI POF is no longer obtained.

Therefore, we modified the interfacial-gel polymerization with several steps. ${ }^{4}$ The two-step interfacialgel polymerization technique is schematically shown in Figure 1. In this process, a thin PMMA homopolymer layer is coated on the inner wall of the MMA3FMA copolymer tube as shown in Figure 1(b). After the polymerization of the PMMA homopolymer layer, the monomer/dopant mixture was injected into the tube, followed by the polymerization of the core region as shown in Figure 1(c). By forming this PMMA homopolymer layer on the inner wall of the tube, the excess scattering is effectively eliminated. The GI POF was obtained by the heat drawing of the GI preform.
In this study the fiber diameter was set to $600 \mu \mathrm{m}$. The effect of the heat-drawing tension on the mechanical strength was investigated in detail as was the case of a PMMA-clad GI POF. ${ }^{4}$ Several GI POFs drawn at the various drawing tensions were obtained from one perform. To vary the drawing tension, the heat-drawing temperature was changed from 180 to $250^{\circ} \mathrm{C}$ during the heat-drawing process. The heat-drawing tension was measured directly by a tension meter during the heat-drawing process. The drawing tension measurement was carried out midway between the bottom of the heat-drawing furnace and the fiber takeup reel.

\section{Evaluation of mechanical strength}

The mechanical strength of the GI POF was investigated based on the measurement method of the tensile strength for the commercially available SI POF., ${ }^{4,6}$ The tensile strengths of the fibers were evaluated by continuously measuring the force that developed as the sample was elongated at a constant rate of extension. The conditions are described below in detail. The measurement apparatus is schematically shown in Figure 2. As shown in Figure 2, both ends of a fiber sample were held between two $3 \times 3-\mathrm{cm}$ plates, and the sample length was approximately $120-130 \mathrm{~mm}$ because the distance from the top of lower plate (II) to the bottom of upper plate (I) was set to $100 \mathrm{~mm}$. The sample elongation speed was set to $100 \mathrm{~mm} / \mathrm{min}$. Figure 3 shows a typical stress-strain curve obtained by this tensile strength measurement. From this chart, not only the tensile strength but also the yield stress, elongation at break, and elongation at yield were analyzed.

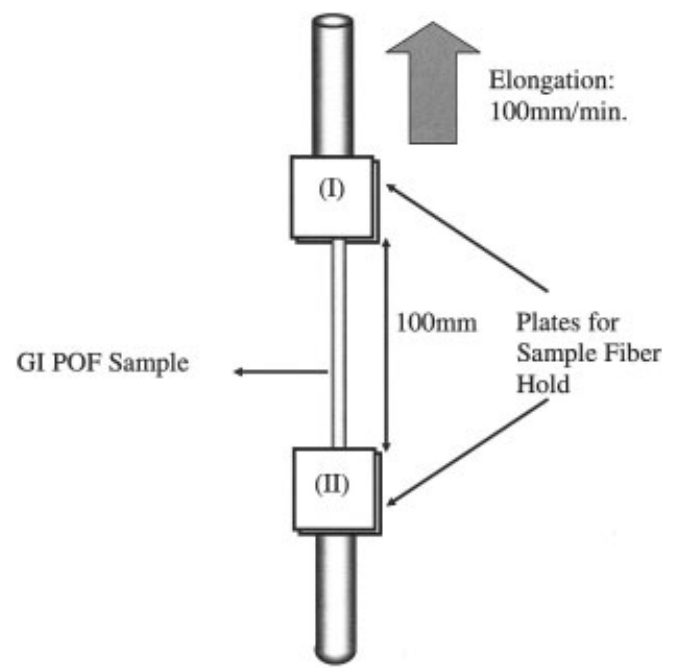

Figure 2 Structure of the GI POF holding part in the mechanical property measurement apparatus. 


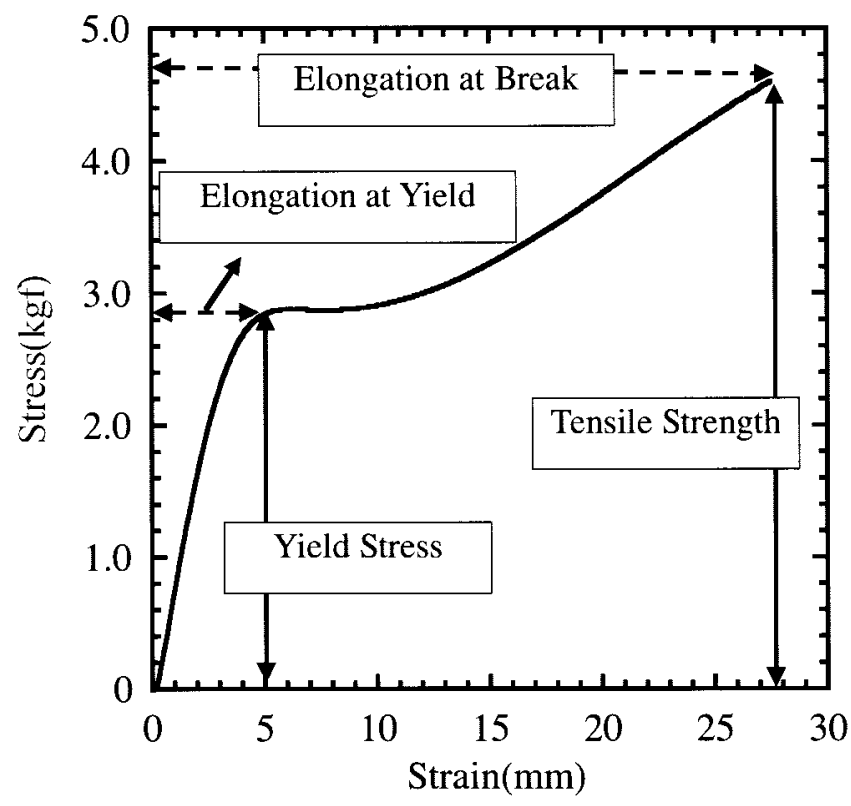

Figure 3 A typical stress-strain curve of the PMMA-based optical fiber and the definitions of several parameters to evaluate the mechanical properties of the GI POF.

\section{RESULTS AND DISCUSSION}

\section{Tensile strength}

The PMMA-based GI POF we have proposed so far is composed of the PMMA homopolymer cladding and of an aromatic compound-doped PMMA core where the dopant material forms a quadratic concentration distribution in the radial direction. This dopant concentration distribution provides the refractive index distribution of the GI POF. To obtain the large refractive index difference between the core and cladding, which offers a high NA, a high dopant concentration is required. For instance, it was shown that an NA higher than 0.2 was necessary for the GI POF to maintain a low bending loss. ${ }^{7}$ To obtain such a high NA in the PMMA-based GI POF, more than $10 \mathrm{wt} \%$ of the dopant concentration is necessary, even if the dopant having a high refractive index such as diphenyl sulfide (DPS) is used. On the other hand, it was previ- ously shown ${ }^{4}$ that if the 3FMA feed ratio to the copolymer cladding was MMA : $3 \mathrm{FMA}=4: 1(\mathrm{wt} / \mathrm{wt})$, the refractive index of the cladding became 1.478 , which is much lower than that (1.492) of the PMMA homopolymer cladding. Therefore, the dopant concentration could be lowered to $5 \mathrm{wt} \%$ in the P(MMA-3FMA)clad GI POF. The refractive indices of the core center and cladding, and the NA of both PMMA-clad and P(MMA-3FMA)-clad GI POFs are summarized in Table I. It is found from Table I that an NA higher than 0.25 could be achieved by only a $5 \mathrm{wt} \%$ doping in the P(MMA-3FMA)-clad GI POF, whereas a 20 wt \% doping is necessary in the PMMA-clad GI POF for the same NA.

The mechanical properties of the GI POFs were evaluated by preparing the PMMA-clad and P(MMA3FMA)-clad GI POFs with 11 and 5 wt \% dopants, respectively. Before evaluating the mechanical properties of both types of GI POFs, the effect of the P(MMA-3FMA)-cladding on the tensile strength was investigated first. For this measurement, the P(MMA3FMA)-clad and PMMA-clad POFs that include no dopant in them were prepared.

Figure 4 shows the relation between the heat-drawing tension and the tensile strength of the P(MMA3FMA)-clad POF compared to that of PMMA homopolymer-clad POF, both of which include no dopant. With increasing heat-drawing tension, the tensile strengths of both the PMMA-clad and $\mathrm{P}$ (MMA-3FMA)-clad POFs also increase. This is because the polymer chains composing the POF are more oriented in its axial direction if it is drawn at a high tension. Therefore, when the POFs were further elongated in the axial direction in the tensile strength measurement, these oriented polymer chains support the fiber against breakage. It is also found from Figure 4 that the use of a P(MMA-3FMA) copolymer for the cladding caused a slight decrease in the tensile strength of the POF. This is because the polymer molecular interactions are weak in the P(MMA-3FMA) copolymer. Because the side chain of the 3FMA polymer has a more bulky structure such as trifluoroethyl group, compared to the methyl group in PMMA, less

TABLE I

Comparison of the Fiber Parameters of Both PMMA-Clad and P(MMA-3FMA)-Clad GI POFs

\begin{tabular}{clcccc}
\hline \multicolumn{1}{c}{ Fiber } & \multicolumn{1}{c}{ Dopant } & $n_{\text {core }}$ & $n_{\text {clad }}$ & $\Delta n$ & NA \\
\hline P(MMA-3FMA)-clad & DPSO (5 wt \%) & 1.500 & 1.478 & 0.022 & 0.257 \\
& DPS (5 wt \%) & 1.500 & & 0.022 & 0.257 \\
PMMA-clad & DPSO (11 wt \%) & 1.503 & 1.492 & 0.011 & 0.182 \\
& DPS (11 wt \%) & 1.504 & & 0.012 & 0.190 \\
& DPSO (20 wt \%) & 1.512 & & 0.020 & 0.245 \\
& DPS (20 wt \%) & 1.512 & & 0.020 & 0.245 \\
\hline
\end{tabular}




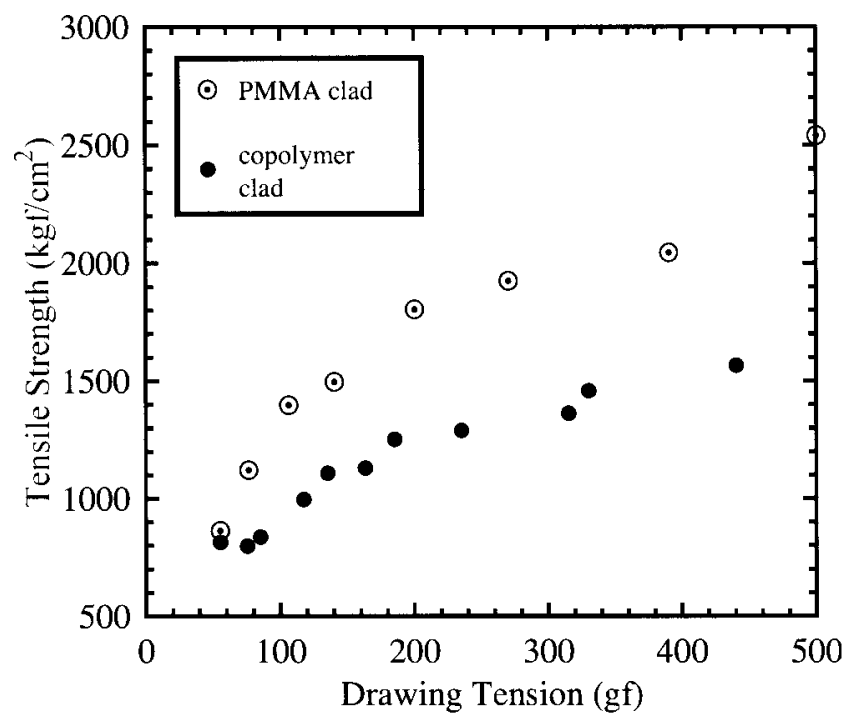

Figure 4 Relation between the heat-drawing tension and the tensile strength of the GI POFs including no dopant.

entanglement among the polymer chains is expected in the P(MMA-3FMA)-clad GI POF. Therefore, if a tensile strength greater than $1000 \mathrm{kgf} / \mathrm{cm}^{2}$ is required for the P(MMA-3FMA)-clad GI POF, a drawing tension higher than $120 \mathrm{gf}$ is also needed, whereas only a 60-gf drawing tension allows the PMMA-clad POF to have a tensile strength greater than $1000 \mathrm{kgf}$. However, as shown in Figure 4, if the P(MMA-3FMA)-clad GI POF is prepared by drawing at a high tension, a tensile strength greater than $1000 \mathrm{kgf} / \mathrm{cm}^{2}$ is easily obtained, which is much higher than that specified in the JIS standard. ${ }^{8}$

\section{Dopant effect on tensile strength}

In addition to the usage of a P(MMA-3FMA) copolymer for the cladding, the aromatic compound used as the dopant might also reduce the mechanical strength of the GI POF, given that some dopants behave as plasticizers. Therefore, the relation between the tensile strength and the heat-drawing tension of the P(MMA3FMA)-clad GI POF doped with 5 wt \% dopant is shown in Figure 5, compared to that of the PMMAclad GI POF. The dopants used in the P(MMA3FMA)-clad GI POF are DPS and diphenyl sulfoxide (DPSO). The tensile strength of the GI POF is almost the same as that of the POF with no dopant added. In the case of a PMMA-clad GI POF, a tensile strength decrease was observed when the dopant concentration was higher than $11 \mathrm{wt} \%$. However, a higher dopant concentration was preferred to increase the NA of PMMA-clad GI POF, by which low bending loss was achieved. On the other hand, the P(MMA3FMA) cladding, with a lower refractive index than that of the PMMA cladding, allows a lower dopant concentration such as $5 \mathrm{wt} \%$ while maintaining a high NA.

\section{Dopant effect on length shrinkage}

There is a concern in drawing the GI POF at a high drawing tension: fiber length shrinkage if the fiber is placed in a high-temperature atmosphere. Because the polymer chains in the GI POF are highly oriented in the axial direction when it was drawn at a high tension, a relaxation of the orientation tends to occur, particularly if the fiber is placed at the temperature close to its glass-transition temperature $\left(T_{g}\right)$. Therefore, the drawing tension effect on the length shrinkage of the P(MMA-3FMA)-clad GI POF at the high temperature was experimentally investigated. Several 1-m pieces of P(MMA-3FMA)-clad GI POFs drawn at various tensions were oven aged at $70^{\circ} \mathrm{C}$ for $48 \mathrm{~h}$; subsequently the resulting fiber length was compared with that before aging to estimate the length shrinkage. The relation between the heat-drawing tension and the length shrinkage after $48 \mathrm{~h}$ of aging at $70^{\circ} \mathrm{C}$ is shown in Figure 6. In Figure 6 the length shrinkage of the PMMA homopolymer POF (including no dopant) is also indicated by the symbol $(\odot)$ as a comparison. It is noted that the length shrinkage of the $5 \mathrm{wt} \%$ doped P(MMA-3FMA)-clad GI POF is almost the same as that of the PMMA homopolymer POF, which is smaller than that of the $11 \mathrm{wt} \%$ doped PMMA-clad GI POF if they are drawn at the same tension. This is because the length shrinkage of the P(MMA-3FMA) polymer fiber (including no dopant) is lower than that of the PMMA homopolymer fiber drawn at the same tension, as shown in Figure 6 by the symbol (@).

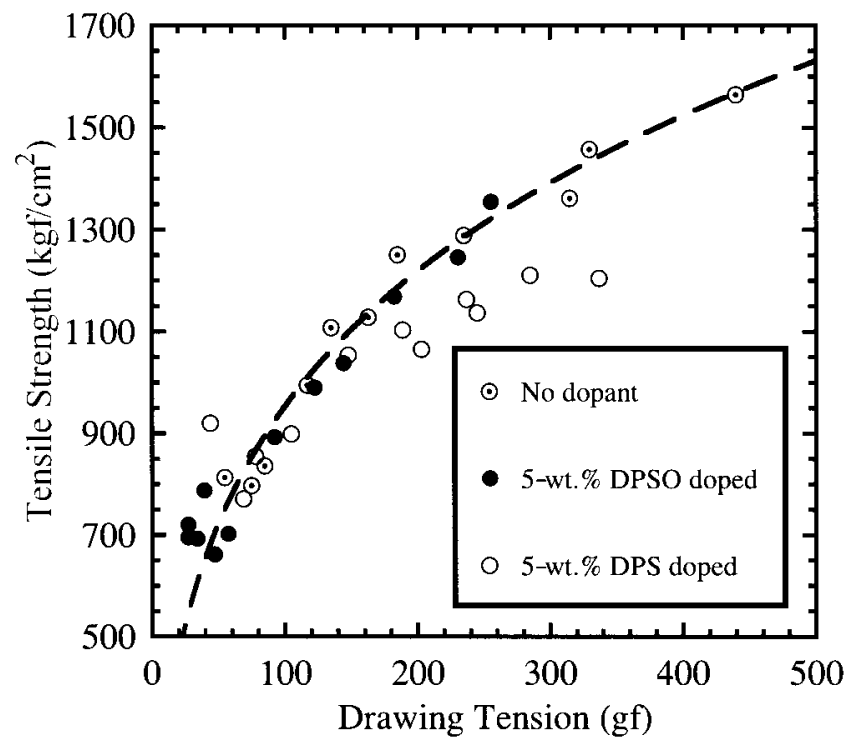

Figure 5 Relation between the heat-drawing tension and the tensile strength of the P(MMA-3FMA)-clad GI POF. 


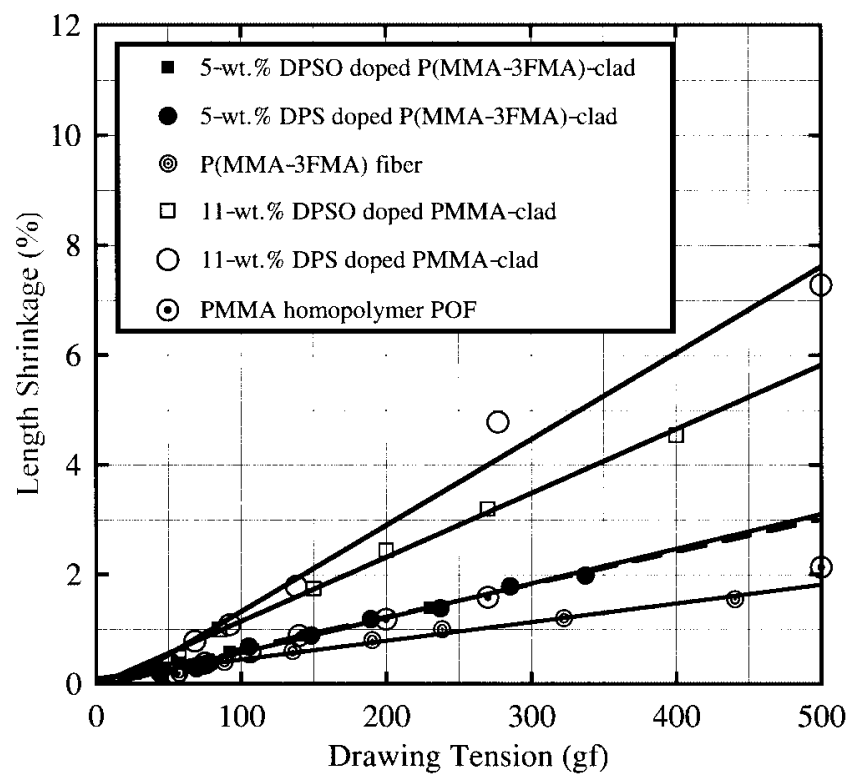

Figure 6 Relation between the heat-drawing tension and the length shrinkage of the GI POFs after 48 -h aging at $70^{\circ} \mathrm{C}$.

Figure 6 shows a maximum of 160-gf drawing tension is allowed in the P(MMA-3FMA)-clad GI POF (5 wt \% doping) while maintaining the length shrinkage of less than $1 \%$. On the other hand, the heat-drawing tension of the PMMA-clad GI POF (11 wt \% doping) should be lower than 80 gf to maintain the length shrinkage lower than 1\%, as shown in Figure 6. Drawing the P(MMA-3FMA)-clad GI POF at such a high tension is also effective for obtaining high tensile strength as mentioned above.

\section{Dopant effect on other mechanical properties}

Because the fiber deformation from no strain state to the yield point, shown in the stress-strain curve in Figure 2, is an elastic deformation, this deformation is reversible. In contrast, the deformation from the yielding to breaking is the viscous deformation; thus it is not a reversible deformation. Therefore, for installing the GI POF cables in office and home networks, the yield stress and elongation should also be the key parameters. The relations between the drawing tension and the yield stress, elongation at yield, and elongation at break of the P(MMA-3FMA)-clad GI POF are shown in Figures 7, 8, and 9, respectively. The results for the P(MMA-3FMA)-based POF (including no dopant) are also indicated in Figures 7, 8, and 9 for comparison. The yield stress of the P(MMA-3FMA)clad GI POF is almost the same as those of the undoped counterparts. These results indicate that the dopant effect on the mechanical properties of a P(MMA-3FMA)-clad GI POF is small because the dopant concentration is lowered to $5 / \mathrm{wt} / \%$.

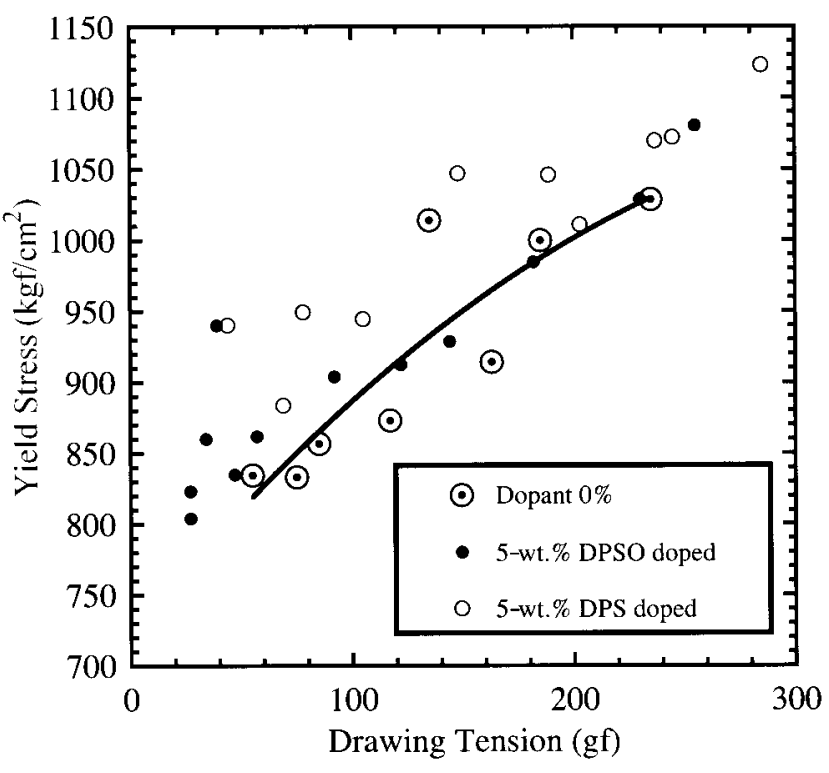

Figure 7 Relation between the heat-drawing tension and the yield stress of the P(MMA-3FMA)-clad GI POF.

Slightly low elongation at yield is observed in the P(MMA-3FMA)-clad GI POF compared with that of the GI POF with no dopant, as shown in Figure 8. This means that the dopant addition lowers the entanglement of polymer chains in the P(MMA-3FMA)-clad GI POF. However, Figure 8 also indicates that the elongation at yield is also changeable by varying the heat-drawing tension. Therefore, the optimization of the heat-drawing tension becomes important. This issue is described in the next section.

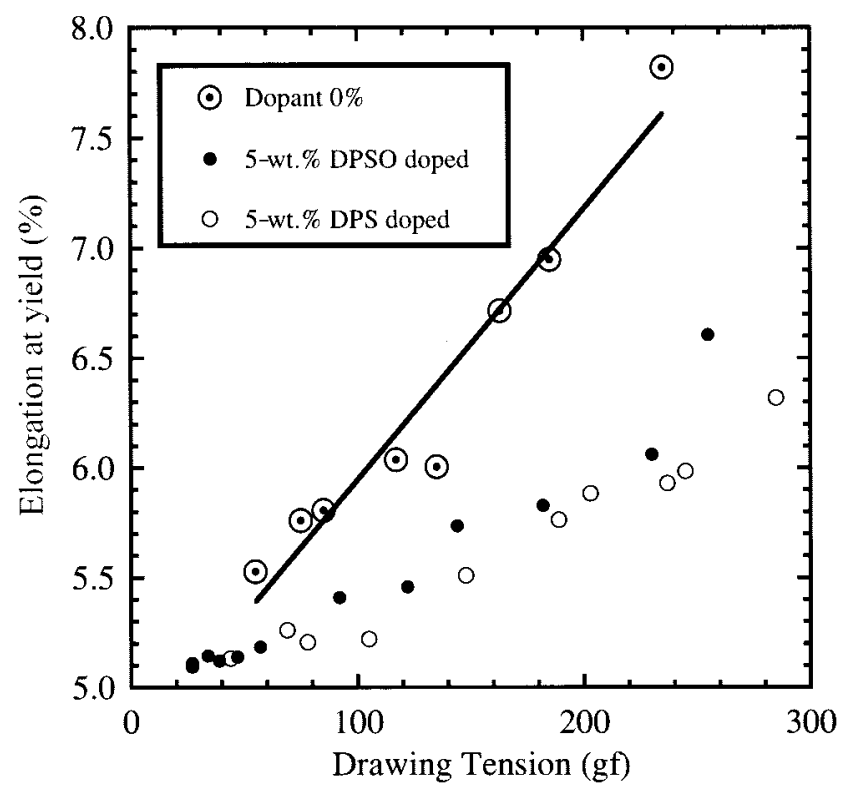

Figure 8 Relation between the heat-drawing tension and the elongation at yield of the P(MMA-3FMA)-clad GI POF. 


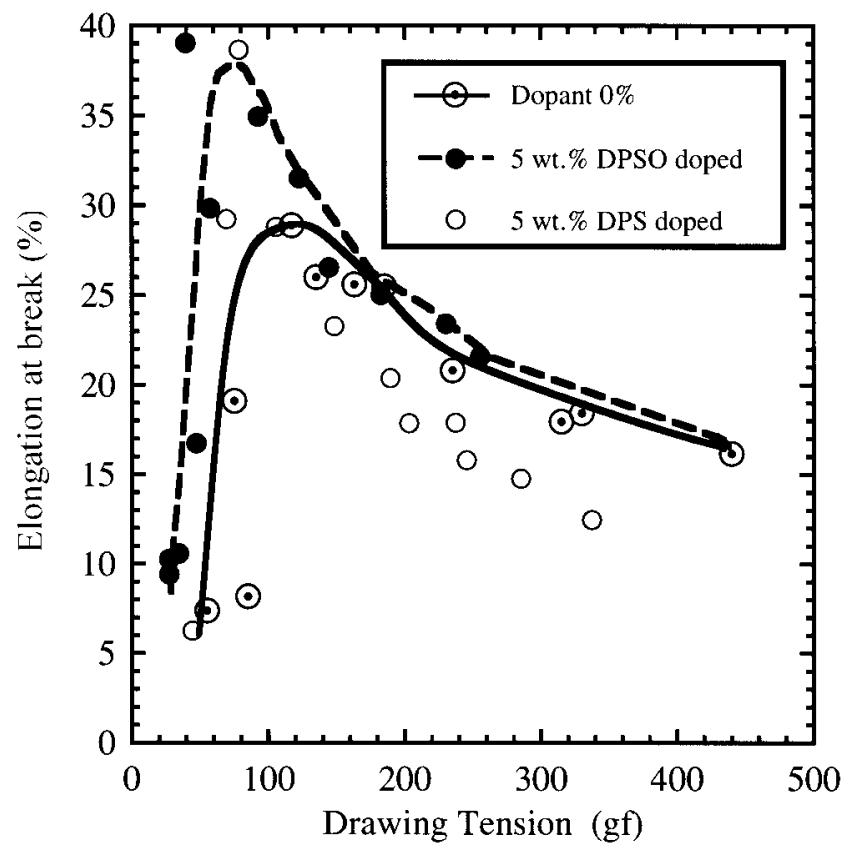

Figure 9 Relation between the heat-drawing tension and the elongation at break of the P(MMA-3FMA)-clad GI POF.

Regarding the elongation at break, the maximum peak is observed when the drawing tension is around 80 gf, which was also shown in the PMMA-clad GI POF. ${ }^{4}$ Furthermore, it is noteworthy that the elongation at break of the $5 \mathrm{wt} \%$ doped P(MMA-3FMA)clad GI POF is slightly larger than that with no dopant, as shown in Figure 9. This phenomenon is called as the "antiplasticization effect"; it is observed when the dopant concentration is small. ${ }^{9}$ This effect will be investigated in detail elsewhere.

\section{Optimum preparation condition of P(MMA- 3FMA)-clad GI POF}

We have shown that the mechanical properties of the P(MMA-3FMA)-clad GI POF are strongly dependent on the heat-drawing tension, whereas little influence of the dopant was observed. In this section, an optimum preparation conditions of the P(MMA-3FMA)clad GI POF is discussed, by which both high mechanical strength and high thermal stability are obtained. At first, the drawing tension should be determined to let the GI POF maintain less than 1\% of the length shrinkage after $48 \mathrm{~h}$ aging at $70^{\circ} \mathrm{C}$. The $1 \%$ length shrinkage is the same value as that of the commercially available SI POF. From Figure 6, the drawing tension is determined to be lower than $160 \mathrm{gf}$ in the case of the 5 wt \% doped P(MMA-3FMA)-clad GI POF. On the other hand, with decreasing drawing tension, all the mechanical properties such as tensile strength, yield stress, elongation at break, and elongation at yield are also lowered. Therefore, the minimum drawing tension of the P(MMA-3FMA)-clad GI POF should be also defined. Figure 10 shows the relation between the drawing tension and the yield stress of both the PMMA-clad (11 wt \% DPS doped) and the P(MMA-3FMA)-clad (5 wt \% DPS doped) GI POFs. To determine the minimum drawing tension, the yield stress of the PMMA-clad GI POF achieved at its maximum drawing tension is the target value that should be achieved by the P(MMA-3FMA)-clad GI POF. Figure 6 shows that the maximum drawing tension of the PMMA-clad GI POF was 80 gf because it allowed less than $1 \%$ length shrinkage during aging. Next, the yield stress achieved by the PMMA-clad GI POF drawn at 80-gf drawing tension is found to be 970 $\mathrm{kgf} / \mathrm{cm}^{2}$, shown by the horizontal broken line in Figure 10 . The yield stress of the commercially available SI POF is specified in the JIS standard ${ }^{8}$ to be higher than $728 \mathrm{kgf} / \mathrm{cm}^{2}$. Therefore, this target value $(970$ $\mathrm{kgf} / \mathrm{cm}^{2}$ ) fully satisfies the specified value in the SI POF. Finally, it is determined from Figure 10 that the minimum drawing tension of the P(MMA-3FMA)clad GI POF should be 120 gf by which almost the same yield stress as $970 \mathrm{kgf} / \mathrm{cm}^{2}$ is achieved. Therefore, it was concluded that the optimum drawing tension of the P(MMA-3FMA)-clad GI POF was from 120 to $160 \mathrm{gf}$.

\section{Mechanical properties of the GI POF after aging}

The high mechanical strength of both the PMMA-clad and P(MMA-3FMA)-clad GI POFs is provided by the orientation of polymer chains in the axial direction,

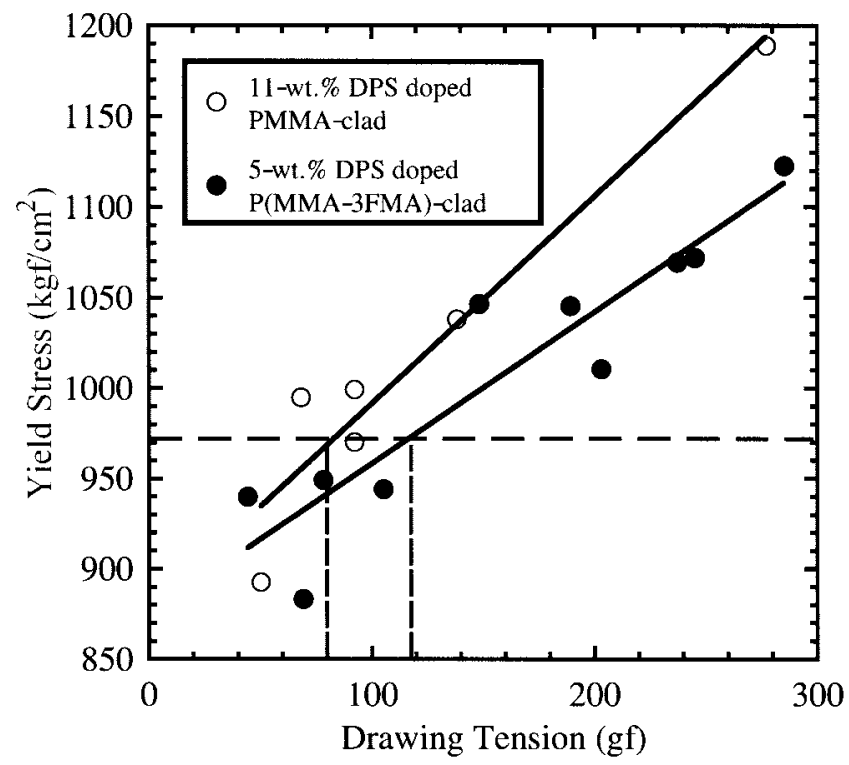

Figure 10 Comparison of the drawing tension dependency of the yield stress of both PMMA-clad and P(MMA-3FMA)clad GI POFs for the definition of the optimum drawing condition. 


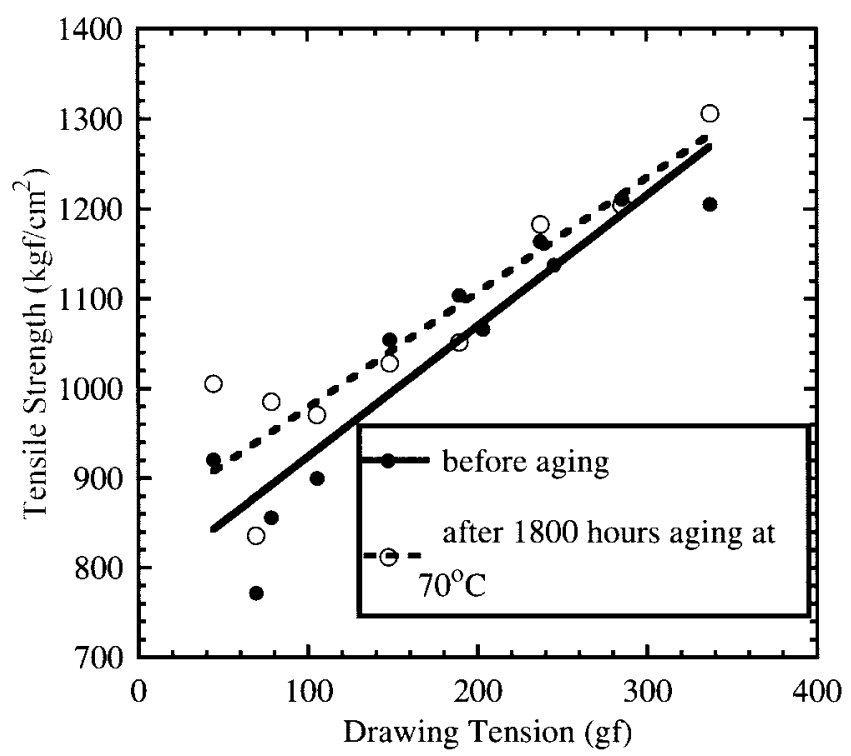

Figure 11 Temperature stability of the tensile strength of the $5 \mathrm{wt} \%$ DPS doped P(MMA-3FMA)-clad GI POF.

formed during the heat-drawing process of the GI POF. It was also shown that the orientation relaxation of the polymer chains occurred if the GI POF was oven aged. Therefore, there is a concern that the mechanical strength of the GI POF might deteriorate after aging because the orientation of the polymer chains would relax during aging. The mechanical strength of the GI POF after aging was evaluated. The tensile strengths of the P(MMA-3FMA)-clad GI POFs were measured before and after $1800 \mathrm{~h}$ of aging at $70^{\circ} \mathrm{C}$. The results are shown in Figure 11. The original tensile strength of the P(MMA-3FMA)-clad GI POF is maintained during aging, even if the drawing tension is higher than the optimum (120-160 gf). It is interesting that the tensile strength after aging is slightly higher than that before aging. It was confirmed that the P(MMA-3FMA)-clad GI POF showed not only excellent optical properties but also good mechanical properties and thermal resistance.

\section{CONCLUSIONS}

The mechanical properties of the newly developed P(MMA-3FMA)-clad GI POF were investigated in detail. Because of the P(MMA-3FMA) cladding, the mechanical strength of the P(MMA-3FMA)-clad GI POF is slightly lower than that of a PMMA-clad GI POF drawn at the same tension. However, because a lower dopant concentration (5 wt \%) is allowed in the P(MMA-3FMA)-clad GI POF compared to the PMMA-clad one while maintaining a sufficient NA, a higher heat-drawing tension could be adopted, by which almost the same or superior mechanical properties are achieved by the P(MMA-3FMA)-clad GI POF compared to the PMMA-clad one. This P(MMA3FMA)-clad GI POF has all of the required properties such as high bandwidth, low loss, low bending loss, high temperature stability, and high mechanical strength. Therefore, we believe that the GI POF will support the enormous capacity in the forthcoming broadband era.

\section{References}

1. Ishigure, T.; Nihei, E.; Koike, Y. Appl Opt 1994, 33, 4261.

2. Koike, Y.; Ishigure, T.; Nihei, E. J Lightwave Technol 1995, 37, 1475.

3. Sato, M.; Hirai, M.; Ishigure, T.; Koike, Y. J Lightwave Technol 2000, 18, 2139.

4. Ishigure, T.; Hirai, M.; Sato, M.; Koike, Y. J Lightwave Technol, to appear.

5. Ishigure, T.; Sato, M.; Takanashi, O.; Nihei, E.; Nyu, T.; Yamazaki, S.; Koike, Y. J Lightwave Technol 1997, 15, 2095.

6. Kitazawa, M. POF Data Book; MRC Technology: Tokyo, 1993.

7. Ishigure, T.; Horibe, A.; Nihei, E.; Koike, Y. J Lightwave Technol 1995, 13, 1686.

8. Test Methods for Mechanical Characteristics of All Plastic Multimode Optical Fibers and Cords, JIS Standard, C6861:1999.

9. Stepek, J.; Daoust, H. Additive for Plastics; Springer-Verlag: Berlin, 1975; Chapter 1. 